\title{
A Review of Primary Immune Deficiency Disorders
}

\author{
Authors: \\ Talin Darian, ${ }^{1}$ Joudeh B. Freij, ${ }^{1}$ Divya Seth, ${ }^{2}$ Pavadee Poowuttikul, ${ }^{2}$ \\ *Elizabeth Secord ${ }^{2}$ \\ 1. Wayne State University School of Medicine, Detroit, Michigan, USA \\ 2. Department of Pediatrics, Division of Allergy, Asthma and Immunology, Children's \\ Hospital of Michigan, Wayne State University, Detroit, Michigan, USA \\ *Correspondence to esecord@med.wayne.edu \\ Disclosure: $\quad$ The authors have declared no conflicts of interest. \\ Received: $\quad 04.03 .20$ \\ Accepted: $\quad 14.05 .20$ \\ Keywords: $\quad$ Agammaglobulinemia, B cell deficiency, hypogammaglobulinemia, innate immune \\ deficiency, newborn screening (NBS), primary immunodeficiency disorders (PIDD), T \\ cell deficiency. \\ Citation: $\quad$ EMJ Allergy Immunol. 2020;5[1]:70-77.
}

\section{Abstract}

This scenario-based review of primary immunodeficiency diseases (PIDD) discusses the differential diagnosis, usual presentations, work-up, and treatment of children with the most commonly encountered immune disorders. Newborn screening (NBS) for severe combined immunodeficiency (SCID) is covered, as are later presenting disorders caused by B cell defects and disorders of the innate immune system.

\section{INTRODUCTION}

Primary immunodeficiency diseases (PIDD) can broadly be described as a group of various defects of the immune system which lead to a variable clinical picture of immune dysregulation. The clinical picture present depends upon the specific immune deficiency, but may involve some combination of recurrent infections, autoimmunity, atopy, lymphoproliferative disorders, or malignancy. Prevalence of PIDD is variable depending on the specific immune deficiency; studies performed in Korea, Sweden, Taiwan, South Africa, and Singapore all found primary antibody deficiencies to be most common, followed by defects in phagocytosis.

Because these diseases are very rare, diagnosing them can be difficult and late diagnosis can lead to a worse prognosis and a delay in life-saving treatment. Further, the same genetic defect can have different manifestations of the disease. ${ }^{2}$ Diagnosis thus requires a thorough knowledge of patient history, a physical exam, and appropriate laboratory tests (which can be nonspecific). There are several guidelines in place to help physicians make a proper diagnosis, such as the Jeffrey Modell Foundation ${ }^{3}$ and the American Academy of Allergy, Asthma, and Immunology. ${ }^{4}$

Given the complexity of PIDD, this review provides a scenario-based review of the major PIDD, stating common presenting symptoms, a differential diagnosis, laboratory tests, and the treatment and management of the most common PIDD.

\section{SCENARIO ONE}

Eli is 9 months-old and has a "constant cold." He has had thick nasal discharge for 2 months and two ear infections that have required antibiotics in 
the last 3 months. He has never been hospitalised. There is no family history of PIDD and his 2-yearold sibling is healthy. Eli was born full-term, and newborn $T$ cell receptor excision circle (TREC) screening for severe combined immunodeficiency (SCID) was normal. He was born at the 50 ${ }^{\text {th }}$ percentile and is now at the $10^{\text {th }}$ percentile.

B-cell defects or deficiencies may manifest at this age as maternal antibodies are waning. The usual transplacental transfer of maternal IgG occurs during the third trimester. A preterm baby may not have received all the usual IgG, but this fullterm baby would have received maternal IgG and should have been making his own at this time.

Transient hypogammaglobulinemia of infancy (THI) is commonly identified in the first year of life but some infants have low IgG until they are several years old. Total Ig is low but specific antibodies are present. ${ }^{5}$ Historically this has been considered a normal variant and some increased incidence of infection may be because of $\mathrm{THI}$ or it may be coincidental.,2 $\mathrm{THI}$ is a diagnosis of exclusion. ${ }^{6}$

Selective serum IgA deficiency is relatively common in children and adults. The estimated prevalence is between one in 200 and one in $1,000.7,8$ IgA deficiency is typically asymptomatic but can present with recurrent respiratory infections from encapsulated organisms and gastrointestinal infection and, although it is controversial, there may by an increased incidence of reaction to blood transfusions because of $\lg G$ antibodies targeting $\operatorname{lgA} .{ }^{9}$ Selective IgA is thought to be symptomatic only in individuals who cannot make mucosal $\lg \mathrm{A} .{ }^{9}$

IgA may be the first Ig class to fall in the development of common variable immunodeficiency (CVID). IgA deficient patients are, therefore, usually periodically screened for progression to CVID. Typically, diagnosis of IgA deficiency is made after the fourth year of life and requires that other causes of hypogammaglobulinemia and T-cell defects be excluded. ${ }^{9}$ Eli was 9 months-old and the absence of IgA would still be physiologic.

CVID is a primary immunodeficiency that usually presents in the second or third decade of life with increased infections. The estimated prevalence is one in 20,000 to one in 50,000.10,11 CVID can also present with noninfectious sequelae, such as autoimmune disease..$^{10,12,13}$ It is a heterozygous disease affecting B cells. ${ }^{14}$ The most commonly known defects are mutations in the transmembrane activator and calcium modulator, the inducible costimulator receptor, and some defects of the cluster of differentiation (CD)-19 molecule.8,11 These defects all affect late B-cell function, and patients with CVID typically have normal total B cells, but many will have low memory B cells $\left(\mathrm{CD} 19^{+} \mathrm{CD} 27^{+} \lg \mathrm{M}^{-}\right){ }^{14}$ CVID cannot be diagnosed before the age of 4 years and can only be diagnosed after other identifiable causes of hypogammaglobinemia have been excluded. ${ }^{1115}$ According to the diagnostic criteria there should also be a two-standard deviation decrease of $\lg G$ and either $\lg M$ or $\lg A$ and an inability to make specific antibodies. .11, $^{11}$

Agammaglobulinemia, which is most often caused by a mutation in the Bruton's tyrosine kinase gene (BTK), typically presents at around 6-9 months of age when maternal antibody levels decline, and the infant fails to produce antibodies. ${ }^{16,17}$ Recurrent respiratory infections because of encapsulated bacteria, such as Streptococcus pneumoniae and Haemophilus influenzae Type $\mathrm{B}$, are the most common infections. ${ }^{2,11,17}$ Definitive diagnosis requires the presence of hypogammaglobinemia or agammaglobulinemia and $<2 \% \mathrm{CD} 19^{+} \mathrm{B}$ cells in the peripheral blood. $X$-linked agammaglobulinemia (XLA) can also be confirmed by the absence of the BTK gene.11

Mutations in other genes involved in the development of the early B cell can also cause agammaglobulinemia. ${ }^{18}$ These genes include the $\mu$ heavy chain gene (IGHM), $\lambda 5$ (IGLL1), Iga (CD79A), Ig $\beta$ (CD79B), and $B L N K^{9,16}$ The autosomal recessive mutations result in a clinical picture similar to XLA.

The absence of tonsils has been an important finding in XLA research and sometimes aids diagnosis. ${ }^{9,18}$ Eli presented with recurrent upper respiratory infections and recurrent sinusitis, but cases of meningitis, osteomyelitis, and septicaemia because of encapsulated bacteria are common infections, especially in untreated agammaglobulinemia., 2,11, Patients can also be at risk for chronic diarrhoea because of Campylobacter jejuni and Giardia lamblia infection., ${ }^{2,1,19}$ Because agammaglobulinemia patients have a functional T-cell system, 
most viral infections are cleared without any difficulty. However, they are susceptible to enterovirus infection and when the oral poliovirus vaccine was in common usage, it could cause meningoencephalitis in individuals with undiagnosed agammaglobulinemia. ${ }^{2,11}$

Increased autoimmunity can be seen in patients with XLA but has typically been thought to occur at a lower frequency compared to other primary immunodeficiencies. ${ }^{20}$ Patients with agammaglobulinemia have a higher rate of inflammatory conditions such as rheumatoid arthritis or Crohn's disease than the general population. ${ }^{20}$

Some patients present later than the classic 6-9 months because of liberal use of antibiotics for less severe infections. It is important not to dismiss the diagnosis in older children before investigation. It is also important to be aware of XLA cases reported with, what used to be classified as "hypo IgM," normal IgG and IgA but poor specific antibody formation. ${ }^{21}$

Treatment of XLA involves lifetime replacement of Ig intravenously or subcutaneously. ${ }^{2,11}$ Standard dosing requires an initial dose of $400 \mathrm{mg} /$ kg every 3-4 weeks and subsequent dosing individualised to the patient as determined by IgG levels. The goal of treatment is to minimise infections. Aggressive antibiotic therapy needs to be initiated when any infection is suspected. ${ }^{2,11}$ Haematopoietic stem cell transplant (HSCT) is not routinely recommended for patients with $X L A$, despite the wide use in other PIDD, because intravenous IG therapy is so successful and the limited studies on the efficacy of HSCT do not support its use for agammaglobulinemia., ${ }^{2,1}$

Complete blood count with differential and IgG, IgA, and lgM are sent. He has an lgA level <7 mg/ $d L$, an lgG of $107 \mathrm{mg} / \mathrm{dL}$ (lower-limit of normal for this age is $217 \mathrm{mg} / \mathrm{dL}$ ), and lgM is undetectable. On examination it is noted that there is no tonsillar tissue present. Lymphocyte enumeration is ordered. Eli has no CD19 B cells. Genetic testing confirms a defect in Bruton's tyrosine kinase which stops maturation of B cells. Eli will have an excellent prognosis if treatment is begun as soon as possible.

\section{SCENARIO TWO}

Michael is 6 days old and was born full-term via vaginal delivery to a healthy mother with no perinatal complications or prenatal illnesses. He is breastfeeding well. His parents receive a phone call that NBS for SCID is positive. They are asked to bring him to the immunologist office at the local university health centre.

NBS for SCID detects TREC, circular DNA remnants that are byproducts of newly formed naive $T$ cells. $^{22}$ The TREC assay was piloted in 2008 in Wisconsin ${ }^{23}$ and is now utilised for NBS throughout the USA and in some other countries. ${ }^{24,25}$

Most positive TREC screens are from preterm infants who are not yet making competent $T$ cells because of prematurity and/or illness. Some infants have a transient T-cell lymphopenia and severe illnesses not associated with PIDD, which may result in low $\mathrm{T}$ cells. ${ }^{24,25}$

DiGeorge syndrome (DGS), most often because of 22q11.2 chromosomal deletion, is a common cause of a positive TREC. DGS varies and can affect not only the immune system but also cause facial anomalies, cardiac defects, and parathyroid abnormalities. Complete DGS renders the infant with essentially no $T$ cells because of a lack of the thymic tissue necessary for negative and positive selection of $T$ cells for maturation. ${ }^{26}$ Although chest X-ray may be utilised to evaluate for thymic tissue ('sail sign' or thymic shadow), it is not a reliable tool for diagnosis because thymic tissue may be very small or in an ectopic location. ${ }^{26}$ Flow cytometry for T-cell markers is more reliable than a chest X-ray and less invasive than a CT scan for verification of a functioning thymus. Flow cytometry is utilised to enumerate $\mathrm{CD}^{+} \mathrm{T}$ cells in circulation. Mitogen proliferation, especially to phytohemagglutinin ( $\mathrm{PHA}$ ), is utilised to determine if the T cells present are functional. ${ }^{26}$

The preferred treatment for complete DGS is thymic transplant, with survival rates of $58-75 \% .{ }^{26}$ Thymic transplant is not readily available for most patients as it is approved at only a few centres. DGS patients most often have other conditions that must be treated prior to transplant, most commonly unstable congenital heart disease. 
Partial DGS, or incomplete DGS, can also trigger a positive TREC in infants with low but functioning $T$ cells. Recovery of adequate T-cell numbers occurs over time. There is evidence that patients with incomplete DGS are more likely than the general population to develop autoimmune disease later in life, and it is advisable to monitor these individuals. ${ }^{27}$

PIDD other than SCID that have variably low $T$ cells, such as ataxia-telangiectasia or even hypomorphic forms of SCID, may trigger a low TREC. ${ }^{28}$ Omenn syndrome, which is sometimes associated with a heterozygous defect in the rearrangement of the recombination-activating gene (RAG), was often difficult to diagnose before TREC NBS because the infants had a severe eczematous rash, lymphadenopathy, eosinophilia, hepatosplenomegaly, and $T$ and $B$ cells that are present but nonfunctioning, which are now identified by NBS. ${ }^{28}$

Infants with a positive TREC are evaluated to determine the etiology of the low $T$ cells and to determine if the cause is SCID. The morbidity and mortality for SCID transplant is decreased if performed prior to 3 months of age, and prior to any infectious complications of SCID. ${ }^{29}$ Flow cytometry is used to further delineate the number of T-, B-, and natural killer (NK)lymphocytes in infants with a low TREC. 30

Michael's exam reveals a healthy appearing 6-dayold boy with no heart murmur. Family history is negative for PIDD and Michael has no siblings. Laboratory testing is ordered for lymphocyte enumeration and mitogen proliferation. Blood is drawn and held for genetic screening. Breastfeeding is discussed and the mother's cytomegalovirus (CMV) IgG and PCR are sent to the lab.

If flow cytometry supports SCID there are precautions that should be taken for the infant such as protective isolation. Hospitalisation is encouraged by some, but infants can be at home under strict precautions if hospitalisation is not practical. When considering whether or not to hospitalise the infant prior to transplant, it is important to determine if there are other young children in the home and if the parents are able to strictly supervise the infant until definitive treatment of stem cell or bone marrow transplant can be done.
The infant with SCID will not be able to respond to vaccines and will be started on replacement Ig as maternal antibodies wane. They should not receive vaccines, especially live vaccines which may do harm. Other persons in the home should receive the influenza vaccine to avoid endangering the infant. ${ }^{31}$

All mothers should be counselled to stop breastfeeding until their CMV IgG and IgM serology is known. It is controversial to advise breastfeeding if the mother has CMV negative titers. Some would argue that the mother may develop CMV. Viral infections, in particular CMV, remain the most common cause of death for infants with SCID. If mothers are seropositive, they should avoid breastfeeding entirely and the infant should have a CMV PCR performed weekly. 31,32

There are several measures of prophylaxis for infants with SCID. Ig replacement should be started when maternal antibodies wane. Trimethoprim-sulfamethoxazole for Pneumocystis jiroveci pneumonia prophylaxis after all neonatal jaundice is cleared, fluconazole for fungal prophylaxis, and acyclovir for prophylaxis of herpesvirus should be administered. Palivizumab may be given during the respiratory syncytial virus season. If blood products are needed they should be irradiated, leukocyte depleted, and CMV negative. ${ }^{30}$

In a study conducted after initiation of NBS, 19\% of infants with SCID had X-linked SCID, but this type was previously reported to be approximately half of all cases. The same report revealed that other types of SCID are more common than previously believed, and the incidence of SCID is overall much higher than previously reported. ${ }^{24}$ The less prevalent types of SCID were apparently missed prior to the start of routine screening. ${ }^{24,25}$

X-linked SCID is caused by defects in the common $\mathrm{y}$-chain of the IL receptor. Mutations in this gene result in absent $T$ and NK cells. These patients have the typical SCID phenotype, with presentation in the newborn period characterised by recurrent severe infections, chronic diarrhoea, and failure to thrive if not diagnosed via NBS. ${ }^{32,33}$

Janus kinase-3 (JAK3) deficiency is the other $\mathrm{T}^{-} \mathrm{B}^{+} \mathrm{NK}^{-}-\mathrm{SCID}$, but it is inherited in an autosomal recessive fashion. JAK3 encodes for a tyrosine kinase that is coupled to cytokine receptors that are essential for lymphoid cell development. 
This deficiency is virtually indistinguishable from $X$-linked SCID, with the exception that females could be affected. ${ }^{32}$

If flow cytometry shows absent $\mathrm{T}, \mathrm{B}$, and NK cells, suspicion for adenosine deaminase deficiency (ADA) increases. ADA-deficient SCID accounts for $10-15 \%$ of all cases. ${ }^{28}$ ADA-SCID is a purine metabolism disorder that kills lymphocytes by build-up of toxic metabolites. It affects the immune system but is not a genetic defect intrinsic to the immune system. ${ }^{30}$ This type of SCID is usually diagnosed in infancy but $15-20 \%$ are diagnosed later in life as infections occur. ADA can be missed on NBS if lymphocyte counts have not yet fallen when the NBS card is collected, which has prompted some states to add ADA enzyme screening to NBS.

SCID that is T- and B-cell negative may be caused by RAG1 and 2 deficiency. RAG are expressed by Iymphocytes and help to mediate double strand DNA breakage at recombination sites. . $^{28,32,33}$ Inherited in an autosomal recessive pattern, RAG deficiency is one of the defects that can also cause Omenn syndrome. It is sometimes missed prior to NBS for SCID because there are often lymphocytes present which may be of maternal origin or clonally expanded $T$ cells of infant origin that are poorly functioning. ${ }^{33}$ RAG defects can also cause leaky SCID, a less severe form of SCID that is characterised by low, but not absent, T cells. ${ }^{33,34}$

There are now $>50$ genetic defects associated with SCID. Genetic testing is important because of preparation for bone marrow transplant or stem cell transplant, especially if the defect is in the DNA repair genes. ${ }^{32,35}$ If there is a trial open for genetic therapy for the type of SCID identified, the family may opt to apply for that therapy, which may go on to become standard of care in the near future. ${ }^{36}$

The laboratory results reveal normal absolute count of $B$ cells, but nearly absent $C D 3^{+} T$ cells and absent CD3-CD56 ${ }^{+} \mathrm{CD} 16^{+} \mathrm{NK}$ cells. The few $T$ cells present are predominantly CD45RO', indicating memory cells of maternal origin. Mitogen proliferation to PHA is very low. The genetic screen for this patient is positive for JAK3 deficiency. Gene therapy for JAK3 deficiencies are currently being researched, but no trial is open to this family. Testing is initiated for human leukocyte antigen-matching for bone marrow transplant or stem cell transplant.

\section{SCENARIO THREE}

Hallie is 3 years old and her mother is concerned about mannose binding lectin deficiency (MBL-D), an immune problem that she read about on the internet. Hallie had an abscess in her nappy area about 1 year ago that required drainage and intravenous antibiotic. Her mother believes they told her it was a methicillin-resistant Staphylococcus aureus (MRSA) infection. She has also had several skin abscesses requiring oral antibiotic. She has not had other infections and there is no family history of PIDD. Her umbilical stump separated at 1 week of age. Dentition appears normal. Before lab results are available Hallie develops a fever of $103^{\circ}$ Fahrenheit and shortness of breath and is hospitalised. Physical exam reveals a respiratory rate of 50, hypoxia, and crepitations in the right posterior lung field. A chest X-ray reveals a right lower lobe pneumonia. She is started on antibiotics to cover pneumococcus and community acquired pneumonia.

The complement system is part of the innate immune system, although the classical complement pathway is primarily activated by antigen-antibody complexes. The alternate pathway's main trigger is bacterial endotoxin. Some components of yeast cell wall and mold, typically Aspergillus, also trigger the alternate pathway. The lectin pathway is activated when lectin recognises carbohydrate residues on bacteria and activates the complement system by binding to MBL. Neisseria and Salmonella or yeast are most commonly recognised by MBL. All three complement pathways promote lysis, opsonisation, and phagocytosis of bacteria. ${ }^{37,38}$

Primary complement deficiencies can lead to increased infections and autoimmune disease. C2 component deficiency is one of the most common complement disorders. It can be asymptomatic or can present with sinopulmonary infections or bacterial meningitis. About $10 \%$ of patients with C2 deficiency will have autoimmune disease, most commonly lupus erythematosus. ${ }^{39}$ Terminal complement component deficiencies leave patients prone to Neisseria meningitis. ${ }^{39}$ Deficiencies have 
been described in $\mathrm{C} 3$ and $\mathrm{C} 4$ components of complement, and these may present as infection or autoimmune disease. Most complement deficiencies are autosomal recessive, but Properdin deficiency, an alternate pathway deficiency, is X-linked and results in susceptibility to fulminant meningococcal infections. ${ }^{40}$

Complement disorders other than MLB-D are rare. MBL-D affects $5-10 \%$ of the population and is usually asymptomatic. ${ }^{41}$ If MBL-D is present with another condition, such as cystic fibrosis or HIV, it can hasten the primary disease progression or susceptibility. ${ }^{42,43}$ It is controversial whether the defect/deficiency alone can lead to increased infections, but patients with no other disorder than MBL-D have been reported to have increased infections.

There are many secondary causes of staphylococcal abscesses of the skin (e.g., chronic skin conditions such as atopic dermatitis, burns, and trauma). Some PIDD are characterised by presentation with staphylococcal abscesses. Autosomal dominant hyper IgE syndrome is a deficiency of signal transducer and activator of transcription-3 (STAT3), and affects many systems including skin, skeletal, and immune systems. The disorder was characterised long before the genetic defect was discovered but this has allowed earlier recognition of the disease. ${ }^{44,45}$ Patients typically have high serum IgE, elevated eosinophils, frequent fractures and skeletal anomalies, eczematous skin, and frequent staphylococcal and fungal infections. The abscesses are usually deep without active neutrophils. Neutrophil chemotaxis is often poor in patients with STAT3 deficiency. The abscesses must be drained before they are flocculent, and awareness of this issue is key to decreasing morbidity and mortality. ${ }^{46}$ A multisystem scorecard is utilised for initial diagnosis of STAT3 deficiency, and genetic testing confirms the diagnosis. ${ }^{47}$ This patient does not have a high score on the initial evaluation but gets extra points for young age because symptoms worsen with time.

Leukocyte adhesion deficiency (LAD) is another PIDD that can lead to staphylococcal abscesses. $L A D$ is rare and there are several subtypes, all autosomal recessive in inheritance, which lead to increased abscess formation. In the most common subtype, LADI, the neutrophils can exit the bone marrow and reach the circulation but lack the CD18 ( $\beta-2$ integrin-chain) function necessary for extravasation to infected tissues. ${ }^{48-50}$ Patients have a poor ability to form abscesses. Patients with severe LADI often have late umbilical cord separation, omphalitis, and severe periodontal disease. ${ }^{48,51}$ With moderateto-severe LADI, leukocyte counts are elevated because of the inability to extravasate. ${ }^{48}$ There is variability of LADI with extreme severe disease presenting in the first weeks of life. Without early intervention of stem cell or bone marrow transplant mortality is high, but some children with less severe disease present in later childhood. ${ }^{48,52}$ Flow cytometry is utilised to detect CD18 on leukocytes. ${ }^{50}$

LADII, a glycosylation disorder, is characterised by cognitive deficiency and severe growth failure. ${ }^{53,54}$ LADIII, an integrin activating protein disorder, is associated with a severe bleeding disorder caused by platelet dysfunction. ${ }^{55}$ Neither LADII or LADIII seem likely in Hallie's case.

Chronic granulomatous disease (CGD) is a disorder of the NADPH oxidase system which usually forms hydrogen peroxide $\left(\mathrm{H}_{2} \mathrm{O}_{2}\right)$ and reactive chloride species. ${ }^{56,57}$ Patients are prone to infection with catalase positive organisms such as Staphylococcus, Serratia, and Aspergillus. ${ }^{58,59}$ NADPH oxidase is formed of multiple subunits and there are several known defects in these subunits, the most common being a defect in the gp91 phox unit, which is an X-linked mutation. Some female carriers of this mutation have later onset and less severe disease. The autosomal recessive forms lead to the same types of infection. Patients with CGD may also suffer from granulomas that are thought to be secondary to chronic inflammation, which may cause organ obstructions and chronic pain. ${ }^{59,60}$

The most accurate assay for the diagnosis of CGD involves measurement of hydrogen peroxide in phagocytes by dihydrorhodamine reductase. The assay is performed by flow cytometry. Genetic testing for some X-linked and autosomal recessive forms is available. ${ }^{61}$

Her immunology labs return. IgG, IgA, IgM, and IgE are all within normal limits for her age. Her lymphocytes subsets are within normal range by percentage and by absolute counts. Her recall antibodies to pneumococcal and tetanus are 
protective. Her CH5O is 95\% (normal), and her mannose binding lectin is normal. Her oxidative burst by dihydrorhodamine assay is very low, showing a population of granulocytes that do not stimulate and have poor oxidative burst. Antibiotic coverage is changed to include Staphylococcus.

Morbidity and mortality of CGD has improved significantly since studies supporting that staphylococcal prophylaxis treated with trimethoprim-sulfamethoxazole and fungal prophylaxis treated with itraconazole are of benefit.62,63 Shortly before the antimicrobial prophylaxis studies were released, interferon- $y$ was shown to be of benefit in decreasing infection but has exhibited controversial benefit when paired with prophylaxis.59,64,65 Bone marrow or stem cell transplant remains a possibility for CGD patients, particularly those with severe recurrent infection, and gene therapy trials for X-linked CGD is under investigation."
The pneumonia improves and she is placed on staphylococcal prophylaxis as well as fungal prophylaxis prior to discharge. Discussion of possible use of interferon-y is also presented to the family. Genetic testing is sent to verify the type of autosomal recessive CGD.

\section{CONCLUSION}

NBS for SCID now helps identify many severe immune deficiency cases early in life, but providers must still be vigilant for signs and symptoms of PIDD such as infections, autoimmune disease, and failure to thrive. Family history of PIDD is always an indication to investigate for PIDD. As time goes by more defects of the immune system, either humoral, cellular, or innate, are appreciated and defined.

\section{References}

1. Justiz Vaillant AA, Qurie A, "Immunodeficiency," StatPearls, 2020, Treasure Island: StatPearls Publishing. Available at: https://www. ncbi.nlm.nih.gov/books/NBK500027/ Last accessed: 15 May 2020.

2. Picard $\mathrm{C}$ et al. Primary immunodeficiencies: an update on the classification from the International Union of Immunological Societies Expert Committee for primary immunodeficiencies 2015. J Clin Immunol. 2015;35:696-726.

3. Jeffrey Modell Foundation. Available at: http://www.info4pi.org/. Last accessed: 22 May 2020

4. American Academy of Allergy, Asthma, and Immunology. Available at: https://www.aaaai.org/. Last accessed: 22 May 2020

5. Dalal I et al. The outcome of patients with hypogammaglobulinemia in infancy and early childhood. J Pediatr. 1998;133(1):144-6.

6. McGeady SJ, "Transient hypogammaglobulinemia of infancy," Stiehm ER (ed), Stiehm's Immune Deficiencies (2014), Oxford: Elsevier Limited, pp.41-7.

7. Yel L. Selective IgA deficiency. J Clin Immunol. 2010;30(1):10-6.

8. Pan-Hammarström Q, Hammarström L. Mini-review: antibody deficiency diseases. Eur J Immunol. 2008;38:327-33.

9. Yazdani $R$ et al. Selective IgA deficiency: epidemiology, pathogenesis, clinical phenotype, diagnosis, prognosis and management. Scan J Immunol. 2017:85(1):3-12

10. Abolhassani $\mathrm{H}$ et al. A review on guidelines for management and treatment of common variable immunodeficiency. Expert Rev Clin Immunol. 2013;9(6):561-74.

11. Bonilla FA et. al. Practice parameter for the diagnosis and management of primary immunodeficiency. J Allergy Clin Immunol. 2015;136(5):1186-205.

12. Warrier I, Secord EA. Clinical features of pediatric primary immunodeficiency patients at a tertiary care clinic. J Allergy Clin Immunol. 2006;117(2):S287.

13. Argarwal S, Cunnighma-Rundles C. Autoimmunity in common variable immunodeficiency. Curr Allergy Asthma Rep. 2009;9(5):347-52.

14. Ahn S, Cunningham-Rundles C. Role of B cells in common variable immune deficiency. Expert Rev Clin Immunol. 2009;5(5):557-64.

15. Ameratunga R, Woon ST. Perspective: evolving concepts in the diagnosis and understanding of common variable immunodeficiency disorders (CVID). Clin Rev Allergy Immunol. 2019; doi: 10.1007/s12016-019-087656. [Epub ahead of print].

16. Bruton OC. Agammaglobulinemia. Pediatrics. 1952;9:722-8.

17. Ochs HD et al., "Antibody deficiencies," Stiehm ER et al. (eds.),
Immmunologic disorders in infants and children (2004) 5th edition Philadelphia: Elsevier/Sanders, pp. 356-66.

18. Conley ME et al. Genetic analysis of patients with defects in early B-cell development. Immunol Rev 2005;203(1):216-34.

19. Winkelstein JA et al. X-linked agammaglobulinemia: report on a United States registry of 201 patients. Medicine (Baltimore). 2006;85(4):193-202

20. VP Hernandez-Trujillo et al. Autoimmunity and inflammation in $X$-linked agammaglobinemia. J Clin Immunol. 2014;34(6):627-32.

21. Seth $D$ et al. Review of primary immune deficiency disease diagnosis before and after genetic testing. J Allergy Clin Immunol. 2011;127(2):PAB90.

22. Chan K, Puck JM. Development of population-based newborn screening for severe combined immunodeficiency. J Allergy Clin Immunol. 2005;115(2):391-8.

23. Routes JM et al. Statewide newborn screening for severe T-cell lymphopenia. JAMA. 2009;302(22):2465-7.

24. Kwan A et al. Newborn screening for severe combined immunodeficiency in 11 screening programs in the United States. JAMA. 2014;312(7):729-38.

25. Vogel BH et al. Newborn screening for SCID in New York State: 
experience from the first two years. $J$ Clin Immunol. 2014;34:289-303.

26. Mcdonald-Mcginn DM, Sullivan KE. Chromosome 22q11.2 deletion syndrome (DiGeorge syndrome) Velocardiofacial syndrome). Medicine. 2011;90(1):1-18.

27. $X i e ~ Y$ et al. Autoimmune disorder secondary to DiGeorge syndrome: a long-term follow-up case report and literature review. Beijing Da Xue Xue Bao Yo Xue Ban. 2016;48(6):1086-9.

28. Aleman $\mathrm{K}$ et al. Reviewing Omenn syndrome. Eur J Pediatr. 2001;160:718-25.

29. Railey MD et al. Long term clinical outcome of patients with severe combined immunodeficiency who received related donor bone marrow transplants without pre-transplant chemotherapy or post-transplant GVHD prophylaxis. J Pediatr. 2009;155(6):834-40.

30. Dorsey $M$ et al. Treatment of infants identified as having severe combined immunodeficiency by means of newborn screening. J Allergy Clin Immunol. 2017;139(3):733-41.

31. Kelty WJ et al. The role of breastfeeding in cytomegalovirus transmission and hematopoietic stem cell transplant outcomes in infants with severe combined immunodeficiency. J Allergy Clin Immunol Pract. 2019;7(8):2863-5.

32. Buckley RH. Molecular defects in human severe combined immunodeficiency and approaches to immune reconstitution. Ann Rev Immunol. 2004;22:625-55.

33. Jyonouchi S et al.

Immunodeficiencies associated with abnormal newborn screening for $T$ cell and B cell lymphopenia. J Clin Immunol. 2017;37:363-74.

34. Marella V, Maina V. Omenn syndrome does not live by $V(D) J$ recombination alone. Curr Opin Allergy Clin Immunol. 2011;11(6):525-31.

35. Fleisher TA. Transplantation outcomes for severe combined immunodeficiency 2000-2009. Pediatrics. 2015;136(Suppl 3);S276-7.

36. Mamcarz E et al. Lentiviral gene therapy combined with low-dose busulfan in infants with SCID-X1. N Eng J Med. 2019;380:1525-34.

37. Abbas AK et al. Cellular and Molecular Immunology, 9th ed, Oxford: Elsevier, pp. 272-88.

38. Janeway et al. "The complement system and innate immunity," Immunobiology: The Immune System in Health and Disease (2001), New York: Garland Science.

39. Sjoholm AG et al. Complement deficiency and disease. Molecular Immunol. 2006;43(1-2):78-85.

40. van den Bogaard R et al. Molecular characterisation of 10 Dutch properdin type I deficient families: mutation analysis and $\mathrm{X}$-inactivation studies. Eur J Hum Genet. 200;8(7):513-8.

41. U.S. National Library of Medicine (NIH). Genetics Home Reference: Mannose-binding lectin deficiency. Available at: https://ghr.nlm.nih.gov/ condition/mannose-binding-lectindeficiency. Last accessed: 15 May 2020 .

42. Chalmers JD et al. Impact of mannose-binding lectin insufficiency on the course of cystic fibrosis: a review and meta-analysis. Glycobiology. 2011;21(3):271-82.

43. Garred P et al. Susceptibility to HIV infection and progression of AIDS in relation to variant alleles of mannose-binding lectin. Lancet. 1997;349(9047):236-40.

44. Sowerwine KJ et al. Hyper-IgE syndrome update. Ann N Y Acad Sci. 2012;1250(1):25-32.

45. Buckley RH et al. Extreme hyperimmunoglobulinemia $\mathrm{E}$ and undue susceptibility to infection. Pediatrics. 1972;49(1):59-70.

46. Sowerwine KJ et al. Hyper-IgE syndrome update. Ann NY Acad Sci. 2012:1250(1):25-32.

47. Hsu AP et al., "Autosomal dominant hyper IgE syndrome," Adam MP et al. (eds), Gene Reviews ${ }^{\circledR}$ (2012), Seattle: University of Washington.

48. Novoa EA et al. Leukocyte adhesion deficiency-l: a comprehensive review of all published case. J Allergy Clin Immunol Pract. 2018;6(4):1418-20.

49. Bowen TJ et al. Severe recurrent bacterial infections associated with defective adherence and chemotaxis in two patients with neutrophils deficient in a cell, associated glycoprotein. J Pediatr. 1982;101(6):932-40.

50. Anderson DC, Springer TA. Leukocyte adhesion deficiency: an inherited defect in the Mac-I, LFA-I, and pl50,95 glycoproteins. Ann Rev Med. 1987;38:175-94

51. Davies EG et al. Defective immune interferon production and natural killer activity associated with poor neutrophil mobility and delayed umbilical cord separation. Clin Exp Immunol. 1982;50:454-60.

52. Qasim W et al. Allogeneic hematopoietic stem cell transplantation for leukocyte adhesion deficiency. Pediatrics. 2009;123(3):838-40.
53. Etzioni A, Tonetti M. Leukocyte adhesion deficiency II-from A to almost $Z$. Immunol Rev. 2000;178(1):138-47.

54. Dauber A et al. Congenital disorder of fucosylation type 2c (LADII) presenting with short stature and developmental delay with minimal adhesion defect. Hum Mol Genet. 2014;23(11):2880-7.

55. Svensson L et al. Leukocyte adhesion deficiency-III is caused by mutations in KINDLIN3 affecting integrin activation. Nat Med. 2009;15:306-12.

56. Baehner RL, Nathan DG. Leukocyte oxidase: defective activity in chronic granulomatous disease. Science. 1967;155(3764):835-6

57. Bridges RA et al. A fatal granulomatous disease of childhood; the clinical, pathological, and laboratory features of a new syndrome. Am J Dis Child. 1959;97(4):387-408.

58. Marciano BE et al. Common severe infections in chronic granulomatous disease. Clin Infect Dis. 2015;15;60(8):1176-83.

59. Winkelstein JA et al. Chronic granulomatous disease. Report on a national registry of 368 patients. Medicine (Baltimore). 2000;79(3):155 69

60. Martire B et al. Clinical features, longterm follow-up and outcome of a large cohort of patients with chronic granulomatous disease: an Italian multicenter study. Clin Immunol. 2008;126(2):155-64

61. Elloumi HZ, Holland SM. Diagnostic assays for chronic granulomatous disease and other neutrophil disorders. Methods Mol Biol. 2007:412:505-23.

62. Margolis DM et al. Trimethoprimsulfamethoxazole prophylaxis in the management of chronic granulomatous disease. J Infect Dis. 1990;162(3):723-6.

63. Weening RS et al. Continuous therapy with sulfamethoxazoletrimethoprim in patients with chronic granulomatous disease. J Pediatr. 1983;103(1):127-30.

64. Mouy R et al. Long-term itraconazole prophylaxis against Aspergillus infections in thirty-two patients with chronic granulomatous disease. J Pediatr. 1994;125(6):998-1003.

65. Gatlin JI et al. Ezekowitz and the international chronic granulomatous disease cooperative study group. A controlled trial of interferon gamma to prevent infection in chronic granulomatous disease. N Eng J Med. 1991;21(324):509-16. 